\title{
High Incidence of Axillary Web Syndrome among Breast Cancer Survivors after Breast Reconstruction
}

\author{
Hsiu-Chen Huang ${ }^{a}$ Hui-Hua Liu ${ }^{a} \quad$ Li-Yun Yin $^{a} \quad$ Chao-Hui Weng ${ }^{a}$ \\ Chien-Liang Fang ${ }^{b, c}$ Cheng-San Yang ${ }^{b, d}$
}

${ }^{a}$ Department of Physical Medicine and Rehabilitation, Chia-Yi Christian Hospital, Chia-Yi, Taiwan; ${ }^{b}$ Department of Plastic Surgery, Chia-Yi Christian Hospital, Chia-Yi, Taiwan; ' ${ }^{\mathrm{D}}$ Department of Food Nutrition and Health Biotechnology, College of Medical and Health Science, Asia University, Taichung, Taiwan; ${ }^{\mathrm{d} D e p a r t m e n t}$ of Sports, Health, and Leisure, Tatung Institute of Technology, Chia-Yi, Taiwan

\section{Keywords}

Breast cancer · Axillary web syndrome .

Breast reconstruction - Myofascial adhesion

\section{Abstract}

Objective: The aim of this study was to identify if breast reconstruction is a surgical risk factor for axillary web syndrome (AWS) in breast cancer (BC) patients. Methods: The data of 207 patients who have been diagnosed with unilateral BC and who had mastectomy and lymph node dissection were retrospectively reviewed. Information of their clinical and pathological data, whether they had immediate reconstruction and intraoperative radiotherapy, surgical methods, and postoperative complications during the 3 months after their surgery (AWS, lymphedema, seroma, and myofascial adhesion) were collected, and the incidence of AWS was compared between different surgical methods. Results: The overall incidence of AWS was $48.8 \%$ in 207 patients. Of the 22 patients who received reconstruction, 19 developed AWS, yielding an incidence of $86 \%$. Multivariate logistic regression modeling showed that patients who underwent reconstruction had a significantly higher incidence of AWS (odds ratio, 4.74), as did patients with postoperative complication of myofascial adhesion (odds ratio, 7.07). Conclusions: $\mathrm{BC}$ survivors after breast reconstruction are susceptible to AWS, and there is a significant association between myofascial adhesion and AWS. Our results can stimulate further investigation and provide an evidence base for the development of educational guidance for patients who plan to undergo breast reconstruction.

(c) 2019 S. Karger AG, Basel

\section{Introduction}

Axillary web syndrome (AWS), a condition that occurs after the interruption of the axillary lymphatic system, is characterized by "cording," a visible and palpable web of taut tissue in the breast, underarm, medial arm, antecubital space, forearm, or abdominal wall, and is clinically associated with pain and limited shoulder range of motion [1-4] (Fig. 1).

The incidence of AWS, which ranges from 28.1 to $48 \%$, is frequently overlooked and not well defined $[3,4]$. AWS generally develops within 1-5 weeks after surgery 


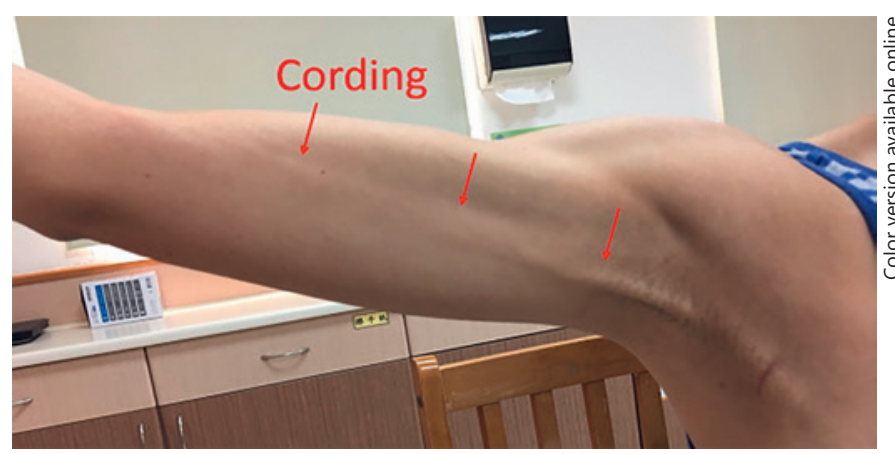

Fig. 1. A 45-year-old female with a palpable cording in the axilla extending down to the elbow after right BC surgery.

and can be resolved within 2-3 months $[4,5]$. Since the cording is more prominent during shoulder abduction, its existence might hinder subsequent treatment by impairing the patients radiotherapy when their arm must be positioned overhead in abduction and external rotation [6-8].

The mechanism of AWS is not yet well established. Koehler [9] related the formation of cording to the healing process - inflammatory, proliferative (angiogenesis), and maturation phases that are in response to the disruption of the lymphatic system and the reconnections of lymph vessels (lymph angiogenesis). Eventually, the newly forming lymphatic vessels may become adhered to the underlying tissue and thus also become palpable to cording.

As advances in breast cancer (BC) treatments increase the patients' survival rate, breast reconstruction becomes a choice for them to improve their body image and the fit of their clothing [10]. Many of the studies previously conducted on breast reconstruction have shown a favorable protective effect on the future development of lymphedema [10-13]. For example, the study by Card et al. [10] revealed that patients who undergo breast reconstruction have a lower incidence and a delay in the onset of BCrelated lymphedema as compared with patients who undergo mastectomy alone.

Among the possible reasons for why reconstruction may be beneficial is that transferred vascularized tissue can bridge damaged lymphatics and reduce scarring following lymph node dissection $[10,11,14-16]$. In addition, vascularized tissue has the potential to restore lymphatic flow by promoting angiogenesis and lymphatic regeneration $[10,17]$.

However, it remains unclear if reconstruction is associated with a higher incidence of myofascial adhesion and/or cording formation. Thus, we have conducted this study to compare the various surgical methods/complications between the patients with and without AWS to identify the risk factors related to surgery for the incidence of AWS.

BC Survivors after Breast Reconstruction Are Susceptible to AWS
Table 1. Patient characteristics

\begin{tabular}{|c|c|c|}
\hline \multicolumn{3}{|l|}{ Continuous variables } \\
\hline Age, years & 52.2 & 10.3 \\
\hline $\mathrm{BW}, \mathrm{kg}$ & 57.6 & 8.5 \\
\hline $\mathrm{BL}, \mathrm{cm}$ & 156.5 & 5.4 \\
\hline BMI & 23.6 & 3.5 \\
\hline Tumor size, cm & 2.4 & 1.8 \\
\hline Removed soft tissue size, $\mathrm{cm}$ & 12.9 & 5.9 \\
\hline LN dissected, $n$ & 10.1 & 9.6 \\
\hline Operating time, $\min$ & 192.7 & 110.0 \\
\hline \multicolumn{3}{|l|}{ Categorical variables } \\
\hline \multicolumn{3}{|l|}{ Type of mastectomy } \\
\hline $\mathrm{PM}$ & 96 & $46.4 \%$ \\
\hline TM & 111 & $53.6 \%$ \\
\hline \multicolumn{3}{|l|}{ Type of LN dissected } \\
\hline ALND & 87 & $42.0 \%$ \\
\hline SLND & 120 & $58.0 \%$ \\
\hline \multicolumn{3}{|l|}{ Reconstruction } \\
\hline Yes & 22 & $10.6 \%$ \\
\hline No & 185 & $89.4 \%$ \\
\hline \multicolumn{3}{|l|}{ IORT } \\
\hline Yes & 35 & $16.9 \%$ \\
\hline No & 172 & $83.1 \%$ \\
\hline \multicolumn{3}{|l|}{ Myofascial adhesion } \\
\hline Yes & 25 & $12.1 \%$ \\
\hline No & 182 & $87.9 \%$ \\
\hline \multicolumn{3}{|l|}{ Seroma } \\
\hline Yes & 14 & $6.8 \%$ \\
\hline No & 193 & $93.2 \%$ \\
\hline \multicolumn{3}{|l|}{ AWS } \\
\hline Yes & 101 & $48.8 \%$ \\
\hline No & 106 & $51.2 \%$ \\
\hline
\end{tabular}

Values are mean and SD or number and percentages. BW, body weight; BL, body length; BMI, body mass index; LN, lymph node; PM, partial mastectomy; TM, total mastectomy; ALND, axillary lymph node dissection; SLND, sentinel lymph node dissection; IORT, intraoperative radiotherapy; AWS, axillary web syndrome.

\section{Materials and Methods}

In adherence to the regulations for Breast Cancer Care Quality Assurance Measures by the Taiwan Ministry of Health and Welfare, we have established a clinical pathway to integrate physical evaluations and therapies in the continuous treatment and care protocol for cancer patients. Each patient with newly diagnosed $\mathrm{BC}$ and on surgical schedule will be referred to the rehabilitation department at time intervals of preoperative assessment and education, immediate postoperative assessment and therapies, 3-4 weeks postoperative assessment and therapies, and before and after chemotherapies and/or radiotherapies. The physical therapy assessment included measurement of shoulder range of motion, muscle strength, grasp power, existence of AWS, lymphedema, seroma, myofascial adhesion, and activities of daily life impairment. To assess AWS, the axilla was palpated, with the shoulder positioned in $90^{\circ}$ of abduction. The patients with AWS were defined as those individuals who have palpable cording from the axilla to the upper limb when positioned with an affected shoulder abduction of $90^{\circ}$ during any point of postoperative assessment within 3 months. 
Table 2. Univariate logistic regression model of the incidence of AWS $(n=207)$

\begin{tabular}{|c|c|c|c|c|c|c|}
\hline Items & \multicolumn{2}{|c|}{ AWS $(n=101)$} & \multicolumn{2}{|c|}{ No AWS $(n=106)$} & Odds ratio $[95 \% \mathrm{CI}]$ & $p$ \\
\hline \multicolumn{7}{|l|}{ Continuous variables } \\
\hline Age, years & 51.1 & 9.7 & 53.2 & 10.7 & $0.98[0.95,1.01]$ & 0.137 \\
\hline $\mathrm{BW}, \mathrm{kg}$ & 56.2 & 8.6 & 59.0 & 8.2 & $0.96[0.93,0.99]$ & $0.018^{*}$ \\
\hline $\mathrm{BL}, \mathrm{cm}$ & 156.4 & 4.9 & 156.6 & 5.9 & $1.00[0.95,1.05]$ & 0.832 \\
\hline BMI & 23.0 & 3.3 & 24.1 & 3.6 & $0.91[0.83,0.98]$ & $0.018^{*}$ \\
\hline Tumor size, $\mathrm{cm}$ & 2.5 & 2.3 & 2.3 & 1.2 & $1.08[0.91,1.27]$ & 0.390 \\
\hline Removed soft tissue size, $\mathrm{cm}$ & 13.3 & 5.5 & 12.4 & 6.2 & $1.03[0.98,1.08]$ & 0.270 \\
\hline LN dissected, $n$ & 10.4 & 10.5 & 9.8 & 8.8 & $1.01[0.98,1.04]$ & 0.635 \\
\hline Operating time, $\min$ & 221.4 & 136.1 & 165.4 & 67.3 & $1.01[1.00,1.01]$ & $0.001^{* *}$ \\
\hline \multicolumn{7}{|l|}{ Categorical variables } \\
\hline \multicolumn{7}{|l|}{ Type of mastectomy } \\
\hline $\mathrm{PM}$ & 40 & $41.7 \%$ & 56 & $58.3 \%$ & Referent & \\
\hline TM & 61 & $55.0 \%$ & 50 & $45.0 \%$ & $1.71[0.98,2.97]$ & 0.057 \\
\hline \multicolumn{7}{|l|}{ Type of LN dissected } \\
\hline SLND & 60 & $50.0 \%$ & 60 & $50.0 \%$ & Referent & \\
\hline ALND & 41 & $47.1 \%$ & 46 & $52.9 \%$ & $0.89[0.51,1.55]$ & 0.683 \\
\hline \multicolumn{7}{|l|}{ Reconstruction } \\
\hline No & 82 & $44.3 \%$ & 103 & $55.7 \%$ & Referent & \\
\hline Yes & 19 & $86.4 \%$ & 3 & $13.6 \%$ & 7.96. [2.28, 27.82] & $0.001^{* *}$ \\
\hline \multicolumn{7}{|l|}{ IORT } \\
\hline No & 85 & $49.4 \%$ & 87 & $50.6 \%$ & Referent & \\
\hline Yes & 16 & $45.7 \%$ & 19 & $54.3 \%$ & $0.86[0.42,1.79]$ & 0.690 \\
\hline \multicolumn{7}{|l|}{ Myofascial adhesion } \\
\hline No & 80 & $44.0 \%$ & 102 & $56.0 \%$ & Referent & \\
\hline Yes & 21 & $84.0 \%$ & 4 & $16.0 \%$ & $6.69[2.21,20.28]$ & $0.001^{* *}$ \\
\hline \multicolumn{7}{|l|}{ Seroma } \\
\hline No & 91 & $47.2 \%$ & 102 & $52.8 \%$ & Referent & \\
\hline Yes & 10 & $71.4 \%$ & 4 & $28.6 \%$ & $2.80[0.85,9.24]$ & 0.091 \\
\hline
\end{tabular}

Values are mean and SD or number and percentages. BW, body weight; BL, body length; BMI, body mass index; LN, lymph node; PM, partial mastectomy. TM; total mastectomy; ALND, axillary lymph node dissection; SLND, sentinel lymph node dissection; IORT, intraoperative radiotherapy; AWS, axillary web syndrome. ${ }^{*} p<0.05 .{ }^{* *} p<0.01$.

Through their medical records, we recruited patients who were diagnosed with unilateral BC and had surgical intervention from January 2016 to October 2017 (a partial mastectomy [PM] or total mastectomy [TM], including modified radical mastectomy) and lymph node dissection (ALND or SLNB) at the Ditmanson Medical Foundation of Chia-Yi Christian Hospital. Patients were excluded if they had undergone neoadjuvant chemotherapy or radiotherapy before surgery, were diagnosed with cancer recurrence or another cancer, had incomplete medical records, or were male. The clinical and pathological data were collected through chart reviews of medical, surgical, pathological, and physical therapy records including age, body weight, body length, BMI, maximum tumor size, removed soft tissue size, pathology, whether they had immediate reconstruction and intraoperative radiotherapy (IORT) or not, type of mastectomy (PM/TM), type of lymph node dissection (ALND/SLNB), number of lymph node dissections, operating time, and postoperative complications during the 3 months after surgery (AWS, lymphedema, seroma, and myofascial adhesion).

We expressed the continuous variables as the mean and standard deviation, and the qualitative variables as the percentage. The $\chi^{2}$ test and Student's $t$ test were used to compare the categorical and continuous data. The correlation between variables was examined using the Spearman and Pearson correlation coefficient when appropriate. Predictive effect of variables for AWS was performed by stepwise logistic regression. All statistical tests were conducted using SPSS for windows version 21.0 (SPSS; IBM Corporation, Somers, NY, USA) with a two-tailed significance level of 0.05 .

\section{Results}

We recruited 238 eligible patients diagnosed with unilateral BC and had pre-and post-operative assessments at Ditmanson Medical Foundation Chia-Yi Christian Hospital. After excluding 11 patients who had received neoadjuvant chemotherapy or radiotherapy before surgery, 11 patients who had another cancer or recurring cancer, 8 patients who had incomplete medical records, and 1 male patient, we collected the complete data for 207 patients. Of the 207 participants analyzed, 101 were in the AWS group and 106 were in the control group, yielding an incidence of $48.8 \%$.

Out of the 207 patients, 111 (53.6\%) underwent TM and 96 (46.4\%) underwent PM, 87 of them (42.0\%) underwent ALND and 120 (58.0\%) underwent SLND, and 22 
Table 3. Multivariate logistic regression model of the incidence of AWS $(n=207)$

\begin{tabular}{lll}
\hline Variables & Odds ratio [95\% CI] & $p$ \\
\hline Age, years & $0.99[0.96,1.02]$ & 0.605 \\
BMI & $0.95[0.86,1.05]$ & 0.298 \\
Tumor size, cm & $1.03[0.83,1.29]$ & 0.773 \\
Type of mastectomy & Referent & \\
$\quad$ PM & $1.71[0.78,3.73]$ & 0.178 \\
$\quad$ TM & Referent & \\
Type of LN dissected & $0.82[0.42,1.58]$ & 0.545 \\
$\quad$ SLND & Referent & \\
$\quad$ ALND & $4.74[1.17,19.17]$ & $0.029^{*}$ \\
Reconstruction & & \\
$\quad$ No & Referent & \\
$\quad$ Yes & $1.59[0.64,3.98]$ & 0.318 \\
IORT & Referent & \\
$\quad$ No & $7.07[2.10,23.81]$ & $0.002^{* *}$ \\
$\quad$ Yes & & \\
Myofascial adhesion & Referent & 0.056 \\
$\quad$ No & $3.46[0.97,12.36]$ & \\
Yes & & \\
Seroma & No &
\end{tabular}

BMI, body mass index; LN, lymph node; PM, partial mastectomy; TM, total mastectomy; ALND, axillary lymph node dissection; SLND, sentinel lymph node dissection; IORT, intraoperative radiotherapy. ${ }^{*} p<0.05$. ${ }^{* *} p<0.01$.

(10.6\%) underwent reconstruction while 35 (16.9\%) underwent IORT. Among the 22 patients with breast reconstruction, there were 19 autologous tissues (16 deep inferior epigastric perforator and 3 transverse rectus abdominis myocutaneous flaps) and 3 implants. The average age of the patients was 52.2 years, while the average BMI at surgery was 23.6 (Table 1 ).

Out of the 22 patients who received reconstruction, 19 developed AWS, yielding an incidence rate of $86 \%$. Univariate logistic regression modeling showed the odds ratio of AWS being associated with reconstruction as 7.96 (95\% confidence interval [CI], 2.28-27.82; $p=0.001)(\mathrm{Ta}-$ ble 2). Patients with postoperative complication of scar tissue adhesion had a greater than six-fold higher incidence of AWS as compared with patients without (odds ratio, 6.69; 95\% CI, 2.21-20.28; $p=0.001$ ) (Table 2). Other risk factors of AWS included body weight (BW), BMI, and operation time (Table 2).

To prevent bias from including high correlation variables $(r \geq 0.7)[18]$ in multivariate logistic regression model, we analyzed the correlation of variables related to AWS. $\mathrm{BMI}$ is defined as BW divided by the square of body length $(\mathrm{BL})$, and as a result, it had a high correlation with BW $(r=-0.997)$ and BL $(r=0.989)$. The type of mastectomy (PM or TM) was highly correlated with removed soft tis- sue size $(r=-0.797)$. Similarly, the type of lymph node dissection (ALND or SLNB) was highly correlated with the number of lymph node dissections $(r=-0.737)$. Finally, reconstruction surgery costs more time than mastectomy alone, hence why reconstruction was highly correlated with operation time $(r=-0.711)$. Therefore, we excluded BW, BL, removed soft tissue size, number of lymph node dissections, and operating time to identify the risk factors related to surgery for the incidence of AWS. The remaining variables including age, BMI, tumor size, date of assessment, type of mastectomy (PM/TM), type of lymph node dissection (ALND/SLND), IORT, scar tissue adhesion, and seroma were all independent variables for AWS. Multivariate logistic regression modeling showed that patients who underwent reconstruction had a significantly higher incidence of AWS (odds ratio, 4.74), and those with postoperative complication of scar tissue adhesion also had a significantly higher incidence of AWS (odds ratio, 7.07) (Table 3). Breast reconstruction and scar tissue adhesion were significantly associated with AWS.

\section{Discussion}

The major finding of this study is that $\mathrm{BC}$ survivors after breast reconstruction were susceptible to AWS as the incidence was up to $86 \%(19 / 22)$, and there was also evidence of a significantly higher risk of AWS (odds ratio, 4.74) when compared with those who underwent mastectomy alone. Because there were scanty studies examining the correlation between AWS and breast reconstruction, we could only find one related article that investigated the clinical and ultrasonographic characteristics of AWS in 36 patients with surgical BC at time intervals of 2 weeks, 4 weeks, and 3 months postoperatively. Their data revealed a $60 \%(3 / 5)$ incidence of AWS in the patients who underwent breast reconstruction [9], but the case numbers of breast reconstruction was too small to make a comparison.

The mechanism behind the higher incidence of AWS in BC survivors after breast reconstruction remains unclear. Most of the previous research regarding damaged upper-limb lymphatic drainage focused on the correlation between the future development of lymphedema and breast reconstruction, and the procedure of reconstructing vessels was hypothesized to be able to help reconnect lymphatic flow and possibly promote angiogenesis and lymphatic regeneration [9-17]. On the other hand, the formation of cording might be a result of the reconnection of damaged lymph vessels (lymph angiogenesis). We speculate that breast reconstruction may help drain the blockage of lymphatics and thus prevent lymphedema, whereas the newly forming lymphatic vessels may cause the formation of cording in the healing process. This hypothesis warrants further investigation. 
We also found a significant correlation between myofascial adhesion and AWS (odds ratio, 7.07). In addition, myofascial adhesion and reconstruction were independent risk factors for AWS because of a low interaction effect $(r=-0.089)$. We speculate that myofascial adhesion caused by scar fibrosis may lead to obstruction of lymphatic drainage, thus making it subject to the formation of cording.

The overall incidence rate of AWS was $48.8 \%$ in our study. In the review article by Yeung et al. [8], the incidence of AWS ranged from $0.6 \%$ to $85.4 \%$, depending on the timing and methods of the assessment and was frequently overlooked. We diagnosed patients with AWS by expert physical therapists during the routine physical assessment, and the incidence rate of $48.8 \%$ was similar to the incidence rate of $47.2 \%$ in the study conducted by Koehler [9] who used similar diagnostic methods. Physiotherapy has been reported to improve shoulder range of motion, to reduce pain, and to shorten cording resolution time $[8,19,20]$. Given the high incidence rate of AWS among BC survivors after breast reconstruction, we advocate for preoperative education about the possibility of developing signs and symptoms of AWS as well as the integration of postoperative assessment and management into the $\mathrm{BC}$ treatment protocol. Furthermore, since cording restricts the range of motion of shoulder abduction [4], medical professionals should plan subsequent radiotherapy with the impact of AWS in mind.

To our knowledge, this is the first study to investigate the correlation between AWS and breast reconstruction. The results from this study could serve as an educational guidance for patients who plan to undergo breast reconstruction and stimulate further investigation.

\section{Conclusion}

$\mathrm{BC}$ survivors after breast reconstruction are susceptible to AWS, and there is a significant correlation between myofascial adhesion and AWS. This is the first study to investigate the correlation between AWS and breast reconstruction. Our results provide a significant evidence base for developing educational guidance for those patients who plan to undergo breast reconstruction and stimulate further investigation.

\section{Acknowledgement}

We thank Guo How-Ran, Ph.D., at the Department of Occupational and Environmental Health, National Cheng Kung University, for providing suggestions regarding statistics and a critical review of the proposal for this study.

\section{Statement of Ethics}

The local ethical committee of Chia-Yi Christian Hospital approved this study in accordance with the Declaration of Helsinki. The study was performed under the Nos. 106008 and 2018004 from the local Ethics Committee of Chia-Yi Christian Hospital.

\section{Disclosure Statement}

The authors declare that they have no competing interests. We declare that no party with a direct interest in the results of the research supporting this article has or will confer benefits to us or any organization we are associated with.

\section{Funding Sources}

We thank Chia-Yi Christian Hospital in Taiwan for the financial support (grant No. R106-18).

\section{Author Contributions}

H.-C.H. conceived of the study, participated in its design and coordination, and drafted the manuscript. C.-H.W. participated in the design of the study and performed the statistical analysis. H.-H.L. and L.-Y.Y. were the main contributors to the data acquisition. C.-L.F. and C.-S.Y. participated in the interpretation of the data and helped draft the manuscript. All authors read and approved the final manuscript.

\section{References}

1 Ferreira Rezende L, Laier Franco R, Costa Gurgel MS. Axillary web syndrome: practical implications. Breast J. 2005 Nov-Dec;11(6):531.

2 Leduc O, Sichere M, Moreau A, Rigolet J, Tinlot A, Darc S, et al. Axillary web syndrome: nature and localization. Lymphology. 2009 Dec;42(4):176-81.

3 Bergmann A, Mendes VV, de Almeida Dias R, do Amaral E Silva B, da Costa Leite Ferreira MG, Fabro EA. Incidence and risk factors for axillary web syndrome after breast cancer surgery. Breast Cancer Res Treat. 2012 Feb; 131(3):987-92.

4 Huang HC, Liu HH, Yin LY, Yeh CH, Tu CW, Yang CS. The upper-limb volumetric changes in breast cancer survivors with axillary web syndrome. Eur J Cancer Care (Engl). 2017 Mar;26(2):e12637.

5 Aydogan F, Belli AK, Bağhaki S, Karabulut K, Tahan G, Uras C. Axillary web syndrome after sentinel node biopsy. Breast Care (Basel). 2008;3(4):277-8.

6 Josenhans E. Physiotherapeutic treatment for axillary cord formation following breast cancer surgery. Z Physiother. 2007;59:868-78.

7 Lattanzi JB, Zimmerman A, Marshall LM. Case report of axillary web syndrome. Rehabil Oncol. 2012;30(1):18-21.

8 Yeung WM, McPhail SM, Kuys SS. A systematic review of axillary web syndrome
(AWS). J Cancer Surviv. 2015 Dec;9(4): 576-98.

9 Koehler LA. Axillary web syndrome ongoing medical evaluation [dissertation]. Minneapolis (MI): University of Minnesota; 2013.

10 Card A, Crosby MA, Liu J, Lindstrom WA, Lucci A, Chang DW. Reduced incidence of breast cancer-related lymphedema following mastectomy and breast reconstruction versus mastectomy alone. Plast Reconstr Surg. 2012 Dec;130(6):1169-78.

11 Chang DW, Kim S. Breast reconstruction and lymphedema. Plast Reconstr Surg. 2010 Jan; 125(1):19-23. 
12 Menezes MM, Bello MA, Millen E, Lucas FA, Carvalho FN, Andrade MF, et al. Breast reconstruction and risk of lymphedema after mastectomy: A prospective cohort study with 10 years of follow-up. J Plast Reconstr Aesthet Surg. 2016 Sep;69(9):1218-26.

13 Miller CL, Colwell AS, Horick N, Skolny MN, Jammallo LS, O'Toole JA, et al. Immediate implant reconstruction is associated with a reduced risk of lymphedema compared to mastectomy alone: a prospective cohort study. Ann Surg. 2016 Feb;263(2):399-405.

14 Chitale VR. Role of tensor fascia lata musculocutaneous flap in lymphedema of the lower extremity and external genitalia. Ann Plast Surg. 1989 Oct;23(4):297-304.
15 Withey S, Pracy P, Wood S, Rhys-Evans P. The use of a lymphatic bridge in the management of head and neck lymphoedema. Br J Plast Surg. 2001 Dec;54(8):716-9.

16 Classen DA, Irvine L. Free muscle flap transfer as a lymphatic bridge for upper extremity lymphedema. J Reconstr Microsurg. 2005 Feb;21(2):93-9.

17 Slavin SA, Van den Abbeele AD, Losken A, Swartz MA, Jain RK. Return of lymphatic function after flap transfer for acute lymphedema. Ann Surg. 1999 Mar;229(3):421-7.
18 Hazra A, Gogtay N. Biostatistics Series Module 6: Correlation and Linear Regression. Indian J Dermatol. 2016 Nov-Dec;61(6):593601.

19 Fourie WJ, Robb KA. Physiotherapy management of axillary web syndrome following breast cancer treatment: discussing the use of soft tissue techniques. Physiotherapy. 2009 Dec;95(4):314-20.

20 Wyrick SL, Waltke LJ, Ng AV. Physical therapy may promote resolution of lymphatic coding in breast cancer survivors. Rehabil Oncol. 2006;24(1):29-34. 